$\mathrm{IC} / 95 / 25$

February, 1995

\title{
$(B+L)$-conserving Nucleon Decays in Supersymmetric Models
}

\author{
Francesco Vissani \\ International Center for Theoretical Physics, ICTP \\ Strada Costiera 11, I-34100 Trieste, Italy \\ and \\ Istituto Nazionale di Fisica Nucleare, INFN \\ Sezione di Trieste, c/o SISSA \\ Via Beirut 2-4, I-34013 Trieste, Italy
}

The presence of the $(B+L)$-conserving decay modes $n \rightarrow$ $K^{+} e^{-}, n \rightarrow K^{+} \mu^{-}, p \rightarrow K^{+} e^{-} \pi^{+}$and $p \rightarrow K^{+} \mu^{-} \pi^{+}$is shown to be a characteristic feature of a class of models with explicit breaking of $R$-parity. These modes dominate over the $(B-L)$ conserving ones in certain regions of the parameter space; the impact of this scenario for nucleon decay search at the SuperKamiokande is discussed.

PACS numbers: 11.30.Fs, 13.30.-a, 12.60.Jv. 
There is a theoretical prejudice in favor of $(B-L)$-conservation in nucleon decay, supported by the following argument. The lowest dimension, gauge invariant operators that give rise to nucleon decay involve necessarily three quarks and one antilepton field [1]. Higher dimensional operators can violate the $\Delta B=\Delta L$ selection rule: this is the case of dimension seven operators that involve three quarks, one lepton and one Higgs-doublet (or one covariant derivative) [1, 2]. If the effective operators are generated by baryonand lepton-number violating interactions at the scale $M_{x}$, it is reasonable to expect that the contribution of the latter to the nucleon decay rate is suppressed by powers of $v / M_{x}$ at best, where $v$ is the scale of $S U(2)_{L}$-symmetry breaking.

Under special conditions the previous argument can be evaded, and ( $B-$ $L$ )-violating decays can reach observable levels: 1) If dimension six operators are not generated. This happens for instance if the four-fermions operators responsible of nucleon decay are induced only after $S U(2)_{L}$-breaking. The four-fermions operators generated by the exchange of a scalar 10-plet in an extension of minimal $S U(5)$ [3] fall in this category. In a different scenario the leading operators for nucleon decay entail six fermions. This has been explicitly realized in a model based on the Pati-Salam Unification group [ [⿴囗十 . 2) If $v / M_{x}$ is not small. The present Letter is devoted to the exploration of this possibility in supersymmetric models. In this context $M_{x}$ will be of the order of the mass of scalar quarks, that we assume in the TeV range.

Let us assume the minimal supersymmetric matter content: one superfield for each ordinary quark and lepton field (for instance the $S U(2)_{L}$-doublet superfield $Q$ corresponds to the the $q_{L}$ quark doublet, and contains the $\tilde{q}_{L}$ scalar quark). Gauge invariance admits the following terms in the superpotential [5] $(R$-parity breaking terms):

$$
\lambda_{i j k} \cdot L_{i} L_{j} E_{k}^{c}+\lambda_{i j k}^{\prime} \cdot D_{i}^{c, \alpha} L_{j} Q_{k}^{\alpha}+\lambda_{i j k}^{\prime \prime} \cdot \epsilon_{\alpha \beta \gamma} D_{i}^{c, \alpha} D_{j}^{c, \beta} U_{k}^{c, \gamma}
$$


where $\alpha, \beta, \gamma$ are color indices, $i, j, k$ are family indices and $S U(2)_{L}$-doublets are contracted by $i \tau_{2}$. It is convenient to consider these couplings in the basis in which $u_{R, i}, d_{R, i}, e_{R, i}, u_{L, i}$ and $e_{L, i}$ are mass eigenstates. Notice that the couplings $\lambda$ and $\lambda^{\prime \prime}$ are antisymmetric in the first two indices.

The exchange of $\tilde{d}_{R, i}$ squarks, in the hypothesis of mass degeneracy, gives rise to the following $(B-L)$-conserving operators [6]:

$$
\frac{2 \lambda_{i l 1}^{\prime \prime}{ }^{*} \lambda_{i j k}^{\prime}}{m_{\tilde{d}_{R}}^{2}} \cdot \epsilon_{\alpha \beta \gamma}\left(d_{R, l}^{\alpha} u_{R}^{\beta}\right)\left(e_{L, j} u_{L, k}^{\gamma}-V_{k n}^{\mathrm{CKM}} \nu_{L, j} d_{L, n}^{\gamma}\right),
$$

where $V^{\mathrm{CKM}}$ is the Cabibbo-Kobayashi-Maskawa matrix. The operators relevant for nucleon decay involve: $e, \mu$ or $\nu_{1}, \nu_{2}, \nu_{3}$ lepton fields; $u$ or $d, s$ quark fields (but no more than one $s$ field). The required suppression of the decay rate is due to the smallness of the $\lambda$-couplings.

New kinds of four fermions operators are induced when the mixing of squarks contained in the $Q$ and in $D^{c}$ (or $U^{c}$ ) supermultiplets is taken into account, since the $B$-violating and $L$-violating interaction terms coming from (II) can be also connected by the $\left\langle\tilde{q}_{R} \tilde{q}_{L}^{*}\right\rangle$ propagator. The induced operators are of the form $(q q)(\bar{l} q)$, and therefore conserve $B+L$; they will be discussed in the following, after recalling the relevant informations on the squark mass matrix.

The $6 \times 6$ matrix for up- or down-type squarks can be written as:

$$
M_{\tilde{q}}^{2}=\left(\begin{array}{cc}
m_{\tilde{q}, L L}^{2} & m_{\tilde{q}, L R}^{2} \\
m_{\tilde{q}, L R}^{2 *} & m_{\tilde{q}, R R}^{2}
\end{array}\right)
$$

To avoid constraints from nonobservation of flavor-changing neutral currents it is usually posited that the off-diagonal elements are small with respect to the diagonal ones. But this condition is not expected to hold for the stop and sbottom left-right mixing terms, since they are proportional to the masses of 
the heaviest quarks:

$$
\begin{aligned}
& m_{\tilde{t}, L R}^{2}=m_{t}\left(A_{t}+\mu \cot \beta\right) \\
& m_{\tilde{b}, L R}^{2}=m_{b}\left(A_{b}+\mu \tan \beta\right)
\end{aligned}
$$

[ $A_{t}$ and $A_{b}$ are the parameters of the Higgs-squark-squark, soft-supersymmetry-breaking interactions; $\mu$ is the supersymmetry-conserving Higgs mass parameter; $\tan \beta$ the ratio of vacuum expectation values $\left.\left\langle H_{u}\right\rangle /\left\langle H_{d}\right\rangle\right]$. For this reason it is important to include the effect of the stop and of the sbottom left-right mixing terms in the discussion.

In the limit of small momenta the propagator $\left\langle\tilde{q}_{R} \tilde{q}_{L}^{*}\right\rangle(q=t, b)$ reduces to

$$
\frac{-i}{\mathcal{M}_{\tilde{q}}^{2}} \equiv \frac{i m_{\tilde{q}, L R}^{2}}{m_{\tilde{q}, L L}^{2} m_{\tilde{q}, R R}^{2}-m_{\tilde{q}, L R}^{4}},
$$

The factor $1 / \mathcal{M}_{\tilde{q}}^{2}$ can be compared with $1 / m_{\tilde{d}_{R}}^{2}$ in eq. (2). The contribution of (5) is enhanced by a large left-right mixing, as well as by a light $\tilde{q}$ squark, since the denominator of the right hand side is the determinant of the stop or of the sbottom mass matrix [7].

The $\tilde{b}_{L}-\tilde{b}_{R}$ exchange gives rise to the following operators:

$$
\frac{\left(2 \lambda_{3 l 1}^{\prime \prime} \lambda_{n j 3}^{\prime}\right)^{*}}{\mathcal{M}_{\tilde{b}}^{2}} \cdot \epsilon_{\alpha \beta \gamma}\left(d_{R, l}^{\alpha} u_{R}^{\beta}\right)\left(\bar{\nu}_{R, j} d_{R, n}^{\gamma}\right),
$$

for which the remarks done for (2) also apply. However this kind of $(B-L)$ violations cannot be experimentally distinguished from a $(B-L)$-conserving signal, since the neutrinos are undetectable.

The case of virtual stop exchange is more interesting. We get the following contributions in the effective lagrangian:

$$
-\frac{\left(2 \lambda_{123}^{\prime \prime} \lambda_{1 j 3}^{\prime}\right)^{*}}{\mathcal{M}_{\tilde{t}}^{2}} \cdot \epsilon_{\alpha \beta \gamma}\left(d_{R}^{\alpha} s_{R}^{\beta}\right)\left(\bar{e}_{R, j} d_{R}^{\gamma}\right),
$$

where $j=1$ corresponds to the electron channel, and $j=2$ to the muon. 
The $\lambda$-couplings appearing in the previous expression differ from those in (2) and in (6). Therefore it is interesting to speculate upon the scenario in which the decay modes induced by (7) are dominating ones. These decay modes are

$$
n \rightarrow K^{+} l^{-}
$$

and

$$
\begin{gathered}
n \rightarrow K^{+} l^{-} \pi^{0} \\
n \rightarrow K^{0} l^{-} \pi^{+} \\
p \rightarrow K^{+} l^{-} \pi^{+},
\end{gathered}
$$

where $l=e, \mu$.

The main contribution to the nucleon decay rate is expected to come from the two-body decays: in fact estimating $\Gamma \sim\left|\lambda^{\prime} \lambda^{\prime \prime}\right|^{2} \Delta m^{5} / \mathcal{M}^{4}$, where $\Delta m$ is the energy release, we obtain $\Gamma$ (three-body) $/ \Gamma$ (two-body) $\sim 15 \%$ for the electron channels, $\sim 7 \%$ for the muon channels. The proton decay rate may be an order of magnitude slower than the neutron three-body decay rates, since it proceeds via the quark-decay mechanism, whereas the neutron decays occur via the more efficient two-quark fusion mechanism (this argument has been proposed in Ref. [8]).

The mode in eq. (8) and the proton decay mode in eq. (9) have already been searched by various experimental groups [9], and the lower bounds on the lifetime translate in the upper bound

$$
\left|\frac{\lambda_{123}^{\prime \prime} \lambda_{1 j 3}^{\prime}}{\mathcal{M}_{\tilde{t}}^{2}}\right| \lesssim 10^{-30} \mathrm{GeV}^{-2},
$$

where $j=1,2$. This bound is saturated by $\left|\lambda_{123}^{\prime \prime} \lambda_{1 j 3}^{\prime}\right| \sim 10^{-26}$, if we assume $\mathcal{M}_{\tilde{t}} \sim 1 \mathrm{TeV}$.

Future search at the Super-Kamiokande [10 may improve the bound (10) by more than an order of magnitude. It is important to remark that the decay 
$n \rightarrow K^{+} l^{-}$provides a quite clear signal in water Čerenkov detectors:

$$
{ }^{16} \mathrm{O} \rightarrow{ }^{15} \mathrm{O}+\gamma(6.2 \mathrm{MeV})+\mu+l,
$$

where $l$ is monochromatic, $\mu$ results from kaon decay and $\gamma$ from the transition of the excited nucleus to the ground state. Since Čerenkov-light detectors cannot distinguish the charges of particles, the signal (11) may be equally well interpreted as the result of the $(B-L)$-conserving $n \rightarrow K^{-} l^{+}$decay. But this two cases are different if one takes into account also other channels. In fact the lowest dimension operator that induces $n \rightarrow K^{-} l^{+}$is of the form $d d u u \bar{s} l$. This implies that the channels $p \rightarrow \bar{K}^{0} l^{+}$and $p \rightarrow \bar{K}^{0} l^{+} \pi^{0}$ are opened as well. Moreover, if six-fermions operators dominate, there is no a-priori reason to expect the suppression of the strangeness-conserving nucleon decays. Instead no other two-body channel is opened in the $(B+L)$-conserving scenario for nucleon decay proposed in the present Letter.

We conclude that the stop-mediated nucleon decays are well characterized at the Super-Kamiokande. This result motivates the experimental search of the kind of signals described above and the theoretical search of a natural framework for small $R$-parity violating couplings.

I would like to thank A. Salam for hospitality at the Centre; A. Yu. Smirnov for invaluable help; G. Senjanović, B. Brahmachari, S. Bertolini, F. Hussain, and G. Thompson for interesting discussions.

\section{References}

[1] S. Weinberg, Phys. Rev. Lett. 43, 1566 (1979); F. Wilczek and A. Zee, Phys. Rev. Lett. 43, 1571 (1979).

[2] S. Weinberg, Phys. Rev. D 22, 1694 (1980); H. A. Weldon and A. Zee, Nucl. Phys. B 173, 269 (1980). 
[3] F. Wilczek and A. Zee, Phys. Lett. B 88, 311 (1979).

[4] J. Pati, A. Salam and U. Sarkar, Phys. Lett. B 133, 330 (1983).

[5] S. Weinberg, Phys. Rev. D 26, 287 (1982); N. Sakai and T. Yanagida, Phys. Lett. B 197, 533 (1982).

[6] I. Hincliffe and T. Kaeding, Phys. Rev. D 47, 279 (1993); Y. Nir and V. Ben-Hamo, Phys. Lett. B 339, 77 (1994).

[7] For low values of $\tan \beta$, in the approximation of M. Carena, M. Olechowski, S. Pokorski and C.E.M. Wagner, Nucl. Phys. B 419, 213 (1994), we find that $\left|1 / \mathcal{M}_{\tilde{t}}^{2}\right|$ is larger than $1 / m_{\tilde{d}_{R}}^{2}$ for gluino masses $\lesssim 500 \mathrm{GeV}$ (neglecting the $\mu \cot \beta$ contribution to $\left.m_{\tilde{t}, L R}^{2}\right)$. For large values, $\tan \beta \gtrsim m_{t} / m_{b}$, also $\left|1 / \mathcal{M}_{\tilde{b}}^{2}\right|$ can be of the same order of magnitude.

[8] C. Jarlskog and F.J. Ynduráin, Nucl. Phys. B 149, 29 (1979).

[9] Fréjus Collab., Ch. Berger et al., Phys. Lett. B 269, 227 (1991); PDG, Phys. Rev. D 50, 1173 (1994).

[10] Y. Totsuka, Report no. ICRR-277-90-20, 1990 (unpublished). 